\title{
BMJ Open Clinical impact of neoadjuvant treatment in resectable pancreatic cancer: a systematic review and meta-analysis protocol
}

Jong-chan Lee, ${ }^{1}$ Soyeon Ahn, ${ }^{2}$ Kyu-hyun Paik, ${ }^{1}$ Hyoung Woo Kim, ${ }^{1}$ Jingu Kang, ${ }^{1}$ Jaihwan Kim, ${ }^{1}$ Jin-Hyeok Hwang ${ }^{1}$

To cite: Lee J-chan, Ahn S, Paik K-hyun, et al. Clinical impact of neoadjuvant treatment in resectable pancreatic cancer: a systematic review and meta-analysis protocol. BMJ Open 2016;6:e010491. doi:10.1136/bmjopen-2015010491

- Prepublication history and additional material is available. To view please visit the journal (http://dx.doi.org/ 10.1136/bmjopen-2015010491).

J-CL and SA are co-first author and contributed equally to this work as the first author.

Received 7 November 2015 Revised 15 February 2016 Accepted 1 March 2016

CrossMark

\begin{abstract}
${ }^{1}$ Department of Internal Medicine, Seoul National University College of Medicine, Seoul National University Bundang Hospital, Seongnam, Korea

2Department of Biostatistics, Seoul National University College of Medicine, Seoul National University Bundang Hospital, Seongnam, Korea
\end{abstract}

Correspondence to Dr Jin-Hyeok Hwang; jhhwang@snubh.org

\begin{abstract}
Introduction: Although the only curative strategy for pancreatic cancer is surgical resection, up to $85 \%$ of patients relapse after surgery. The efficacy of neoadjuvant treatment in resectable pancreatic cancer (RPC) remains unclear and there is no systematic review focusing fully on this issue. Recently, two prospective trials of neoadjuvant treatment in RPC were terminated early because of slow recruiting and existing randomised controlled trials (RCTs) have too small sample sizes. Therefore, to overcome probable biases, it would be more reasonable to include both RCTs and non-randomised studies (NRSs) with selected criteria. This review aims to investigate the effect of neoadjuvant chemotherapy (CTx) and chemoradiation therapy (CRT) in RPC using RCTs and specific NRSs.
\end{abstract}

Method and analysis: This systematic review will include conventional RCTs as group I, and quasirandomised controlled trials, non-randomised controlled trials and prospective cohort studies as group II. Two groups will be assessed and analysed separately. Comprehensive literature search will use Medline, Embase, Cochrane library and Scopus databases. Additionally, we will search references from relevant studies and abstracts from major conferences. Two authors will independently identify, screen, include studies, extract data and assess the risk of bias.

Discrepancies will be resolved by consensus with another author. An independent methodologist will categorise and assess NRSs to minimise heterogeneity. In each study group, meta-analysis will be conducted using a random-effect model and statistical heterogeneity will be evaluated using $\mathrm{I}^{2}$-statistics. Publication bias will be visualised with contourenhanced funnel plots and analysed with Egger's test. In group I, cumulative meta-analysis will be considered because the CTx regimen and CRT protocol have changed. The quality of evidence will be summarised using the GRADE (Grading of Recommendations Assessment, Development and Evaluation) approach.

Ethics and dissemination: This review does not use primary data, and formal ethical approval is not required. Findings will be disseminated through peerreviewed journals and committee conferences.
Strengths and limitations of this study

- This is the first systematic review and meta-analysis focusing solely on neoadjuvant therapy in resectable pancreatic cancer.

- Owing to the characteristics of this issue, we will include randomised controlled trials (RCT) and non-randomised studies (NRS's); the RCTs as group I and NRS's as group II will be assessed and analysed separately.

- We will perform cumulative meta-analysis in group I studies because the neoadjuvant chemotherapy regimen and chemoradiation therapy protocols have changed with time,

- In respect of NRS's, an independent and knowledgeable methodologist will be involved in every step of study selection and analysis with NRS-specific assessment tools.

- To date, there is no large phase III randomised controlled trial of this issue.

Trial registration number: CRD42015023820.

\section{INTRODUCTION}

Description of condition

Pancreatic cancer (PC) is the twelfth most common cancer worldwide, with more than 330000 new cases annually. ${ }^{1}$ It is the fourth and fifth leading cause of cancer-related death in the USA and Europe, respectively. ${ }^{2}$ Unlike other solid malignancies, the 5-year survival rate of PC has not improved significantly over the past few decades and is still around $7 \%$, which is the lowest among various solid malignancies. ${ }^{2} 4$ Although the only curative strategy is surgical resection, less than $20 \%$ of patients with PC are eligible for resection at the time of diagnosis. ${ }^{5}$ Moreover, even after curative resection, the 
cumulative rate of locoregional or systemic recurrence is up to $85 \% .^{6}{ }^{7}$ This implies that many PCs develop early micrometastasis, and therefore even 'resectable' PC (RPC) is sometimes regarded as a systemic, not localised, disease.

In this context, many studies on adjuvant treatment in RPC were reported in the past two decades. In terms of adjuvant chemotherapy (CTx), modest survival gain was shown in several landmark trials, ${ }^{6-10}$ whereas the role of adjuvant chemoradiation therapy (CRT) is still controversial. $^{8}{ }^{11}{ }^{12}$ The US National Comprehensive Cancer Network (NCCN) guideline recommends adjuvant CTx and CRT therapy after resection of PC, and the European guidelines (European Society for Medical Oncology (ESMO), European Society of Digestive Oncology (ESDO)) recommend only adjuvant CTx in the same situation. ${ }^{13}{ }^{14}$ However, the median overall survival (OS) of patients with RPC is still less than 25 months, even after adjuvant therapy, and this poor outcome has led to attempts to investigate neoadjuvant treatment.

\section{Description of intervention}

Neoadjuvant therapy is systemic or locoregional treatment that is performed antecedent to surgery. It can be performed as CTx or CRT. Theoretically, neoadjuvant therapy should have several clinical benefits: (1) elimination of possible micrometastasis, (2) improvement of R0 resection rate, (3) identification of patients with aggressive or rapidly metastatic disease before surgery, and (4) increased completion rate of multimodal treatment. ${ }^{15} 16$ Several small group studies have supported these hypotheses with diverse CTx regimen or radiation doses. ${ }^{17-21}$ Critics, on the other hand, have concerns about neoadjuvant therapy: (1) the possibility that initially operable cancer may progress to inoperable status during neoadjuvant therapy, and that patients lose the chance to undergo surgery; and (2) too small sample sizes and underpowered results of previous trials of neoadjuvant therapy. ${ }^{16}{ }^{22}$ Currently, several prospective trials of neoadjuvant CTx are in progress, with plans to complete final data collection in 2018-2019. ${ }^{23-25}$ One prospective trial was completed in January $2015 .{ }^{26}$ Meanwhile, two prospective trials of neoadjuvant CRT were terminated early in 2015 because of slow recruiting. ${ }^{27} 28$

\section{Controversy in neoadjuvant therapy for RPC}

Since the subject is still under debate, US and European guidelines do not currently provide definite recommendation or restrictions for neoadjuvant therapy for RPC. $^{13} 14$ Recently, two systematic reviews with meta-analyses including neoadjuvant therapy for RPC as a subgroup analysis were published. ${ }^{29}{ }^{30}$ They concluded that neoadjuvant treatment has minimal effect on OS and progression-free survival. In contrast, several authors contended that neoadjuvant therapy has concrete evidence of benefit, and can be recommended as an alternative treatment for RPC. ${ }^{112031} 32$ Interestingly, the two articles with opposing views of neoadjuvant therapy were published by the same journal within 3 years. ${ }^{30} 31$

The reasons for this discrepancy can be summarised as follows. First, those two meta-analyses did not focus fully on neoadjuvant therapy in RPC, which can result in the omission of meaningful studies. In fact, several relevant studies found in our pilot search were excluded by those systematic reviews. Moreover, the recent 'decision analysis' by Sharma et $a l^{31}$ although a fine attempt, was not a direct synthesis of data. This implies that more refined research fully focused on neoadjuvant therapy in RPC is needed. Second, as we can see from the two studies terminated early, ${ }^{27}{ }^{28}$ the prospective trials will be difficult to complete. Therefore, with a conventional 'strict scope' of inclusion criteria, a meta-analysis can easily have a publication bias. Of course, that does not mean that any crude attempt to explore 'every nook and corner' strategy can be accepted because this could result in excessive heterogeneity. Therefore, an extended scope of inclusion criteria that encompass specific types of NRS's is needed to minimise publication bias and heterogeneity. Third, neoadjuvant CTx regimens and CRT protocols for RPC have changed in recent decades. Therefore, it is reasonable to include changes in CTx regimens and CRT protocols in data synthesis and meta-analysis.

\section{Why it is important to do this work}

This systematic review and meta-analysis contains several novel features. First, this is the only systematic review and meta-analysis focused solely on neoadjuvant therapy in RPC. Since previous systematic reviews addressing this issue in RPC included only small sample-sized RCTs with discordant pools, we will include both RCTs and specific types of NRS's. Second, as described in detail in the Methods section, we will categorise eligible studies into two groups: only conventional RCTs as group I, and specific types of NRS's as group II. Each group will be synthesised and analysed separately. Third, we will apply cumulative meta-analysis for RCTs in group $\mathrm{I}^{33}$ because treatment strategies have changed recently. Finally, at every step of inclusion, assessment, synthesis and analysis of NRS's in group II, we will apply the recommendations of 'NRS checklists' from the Ottawa Non-Randomized Studies Workshop ${ }^{34}$ and tools formulated by the Non-Randomized Study Methods Group (NRSMG) of the Cochrane Collaboration, ${ }^{35}$ with an independent methodologist who is knowledgeable about this process.

\section{OBJECTIVES}

The aim of this systematic review is to investigate whether neoadjuvant therapy (CTx or CRT) is effective in treating resectable $\mathrm{PC}$.

\section{METHODS AND ANALYSIS}

The methods for this systematic review will be developed according to the recommendations of the Preferred 
Reporting Items for Systematic Reviews and Meta-Analyses (PRISMA) statement (figure 1). ${ }^{36}$ This review protocol has been published in the International Prospective Register of systematic reviews (PROSPERO) with registration number CRD42015023820.

\section{Criteria for considering studies for this review}

Types of studies

We will categorise the eligible trials into two groups according to the study types. Group I will include only conventional RCTs. Group II will include specific types of NRS's, which involve quasi-randomised controlled trials (Q-RCTs), non-randomised controlled trials (NRCTs) and double-armed prospective cohort studies (PCS's). This categorisation is based on the Cochrane handbook recommendations, ${ }^{35}$ and we will examine the 'actual features' of study designs rather than 'labels' of study designs. Other types of NRS's, such as casecontrol studies, cross-sectional studies and controlled before-and-after studies, will be excluded. Each group will be assessed and analysed separately (table 1). An independent methodologist (SA) will perform this categorisation.

\section{Participants}

The participants of this study will be 18 years of age or older, and diagnosed with RPC. We will include patients who received surgical resection for $\mathrm{PC}$ and exclude patients with borderline resectable (BRPC), locally advanced (LAPC) or metastatic PC (MPC). For any studies covering RPC and other stages of PC, we will extract the data for patients with RPC.

In regard to the definition of resectability status, we will basically follow the NCCN criteria as below. ${ }^{13}$ However, an accurate definition of BRPC is still not unified among various studies. Therefore, if the definition of BRPC or LAPC is not clear in a certain study, we will check the full text to determine the selection of that study. Here, we present summarised definitions of RPC, BRPC and LAPC according to the NCCN 2015 guidelines. In the actual selection process of articles, a more detailed guideline in NCCN will be applied.

1. RPC: pancreatic tumour without arterial or venous tumour contact. If there is a $\leq 180^{\circ}$ venous tumour contact without venous contour irregularity, it will be regarded as RPC.

2. BRPC: solid tumour contact with artery of $\leq 180^{\circ}$. If there is $>180^{\circ}$ of contact without involvement of the aorta in body/tail cancer, it will be regarded as BRPC. For venous contact, a solid tumour with a $>180^{\circ}$ contact or venous contour irregularity will be regarded as borderline resectable status.

3. LAPC: solid tumour contact of $>180^{\circ}$ with arteries, and unreconstructible vein due to tumour involvement of occlusion.

In regard to 'potentially resectable' $\mathrm{PC}$, some articles use this expression as a same meaning with only RPC, whereas others use this as a more extended meaning

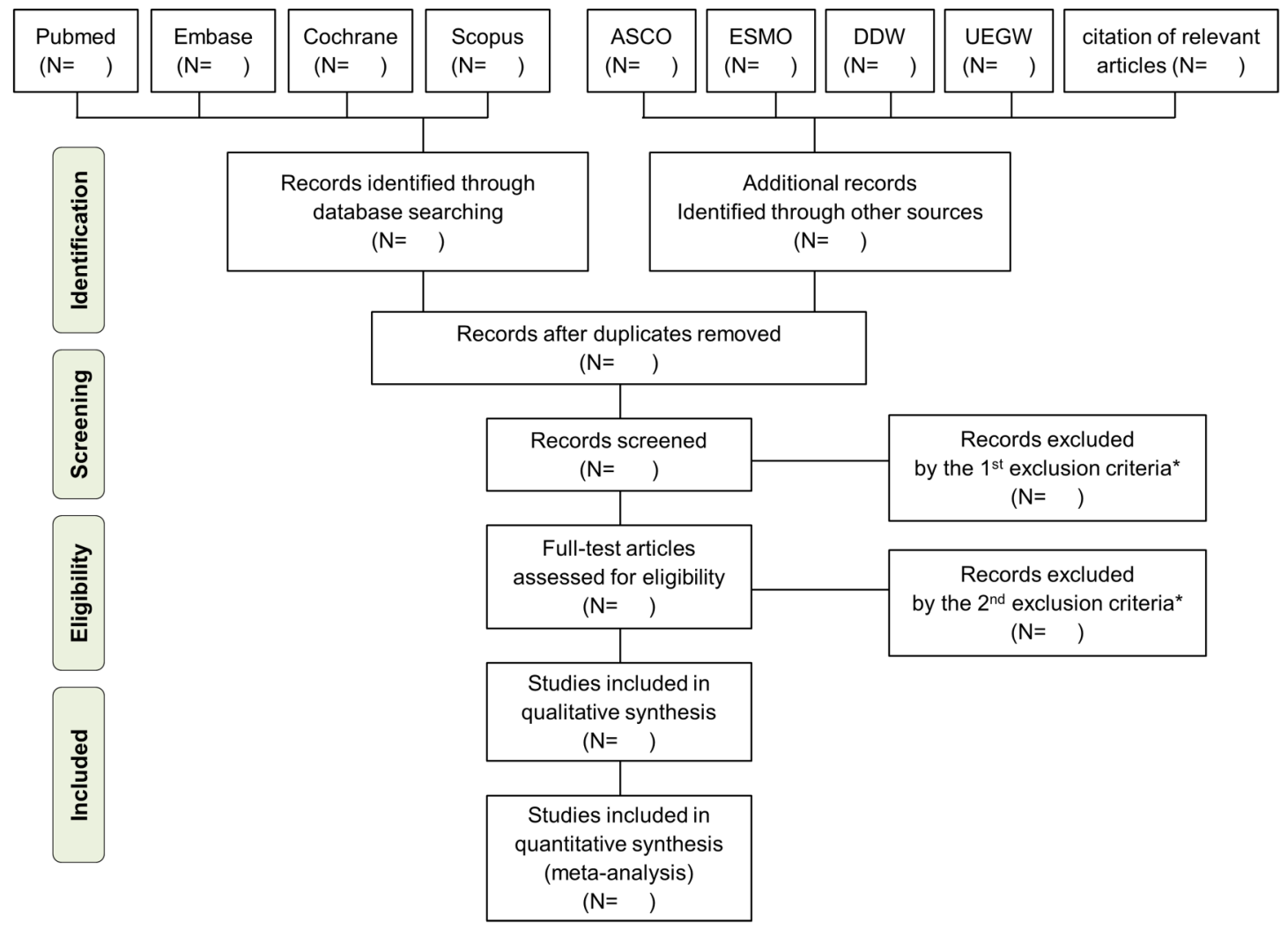

Figure 1 PRISMA flow chart. *The first exclusion criteria, second exclusion criteria and final inclusion criteria for meta-analysis are described in table 3. 
Table 1 Type of studies in two groups

\begin{tabular}{lll}
\hline & Group I & Group II \\
\hline Study types & Conventional RCTs & Q-RCTs \\
& & NRCTs \\
Assessment & $\begin{array}{l}\text { Cochrane Collaboration } \\
\text { risk of bias tool }\end{array}$ & NRS checklist \\
& & Cochrane \\
Collaboration \\
Analysis & Random-effect model & Random-effect \\
& $\begin{array}{l}\text { (DerSimonian-Laird } \\
\text { model }\end{array}$ & $\begin{array}{l}\text { Rect } \\
\text { (DerSimonian- }\end{array}$ \\
& Cumulative & Laird effect) \\
& meta-analysis & \\
\hline
\end{tabular}

non-RCT, non-randomised controlled trials; NRCTs, non-randomised controlled trials; NRSMG, Non-Randomized Study Methods Group; PCS's, double-armed prospective cohort studies; Q-RCTs, quasi-randomised controlled trials; RCT, randomised controlled trials.

including RPC and BRPC. Therefore, we will check the full text of those articles to clarify the inclusion of articles and extraction of data.

\section{Types of intervention and comparator}

The therapeutic intervention includes neoadjuvant CTx and neoadjuvant CRT for RPC. Any CTx regimen and any CRT protocol with information on published years will be included for cumulative meta-analysis. The control group includes the patients who received upfront surgery for PC with or without adjuvant treatment.

\section{Types of outcome measures}

The primary outcomes include OS and disease free survival (DFS) after resection of PC. Since almost RCTs in our pilot search contained the data of OS and DFS, we expect we could perform cumulative meta-analysis and synthesise the survival outcomes. The secondary outcomes include $\mathrm{R} 0$ resection rate because one of the major purposes of neoadjuvant treatment is to improve resectability. If possible, grade III-IV toxicities based on the Common Terminology Criteria for Adverse Events (CTCAE) V.4.03 will be evaluated. ${ }^{37}$ Since the most frequently used adverse event was neutropenia, febrile neutropenia and vomiting, the data of each adverse effect will be extracted.

\section{Data search and selection}

\section{Data sources}

Four electronic databases will be searched from their inception to September 2015: the Cochrane Central Register of Controlled Trials (CENTRAL), Medline (PubMed), Embase and Scopus. In addition, interim analyses from major conferences of the past 10 yearsAmerican Society of Clinical Oncology (ASCO), ESMO, Digestive Disease Week (DDW) and United European Gastroenterology Week (UEGW) - and cited references from relevant articles will be manually searched.

\section{Search strategy}

The global search strategy is shown in table 2 and the detailed strategies for each database are demonstrated in online supplementary appendix 1 . If any up-to-date RCT or NRS is published during the period of this systematic review, we will evaluate the eligibility of the study and consider adding it to the suitable group.

\section{Inclusion and exclusion criteria}

The screening and inclusion will be performed in two steps: first by title and abstract, and second by full-text review. Two independent authors (JL and HK) will conduct this process. Any discrepancies will be resolved in a consensus meeting with a third author (KP) and a methodologist (SA). The detailed inclusion and exclusion criteria are shown in table 3. By using citation programmes such as Endnote, we will create new folders matched to the lists of included and excluded studies.

Table 2 Global search strategy

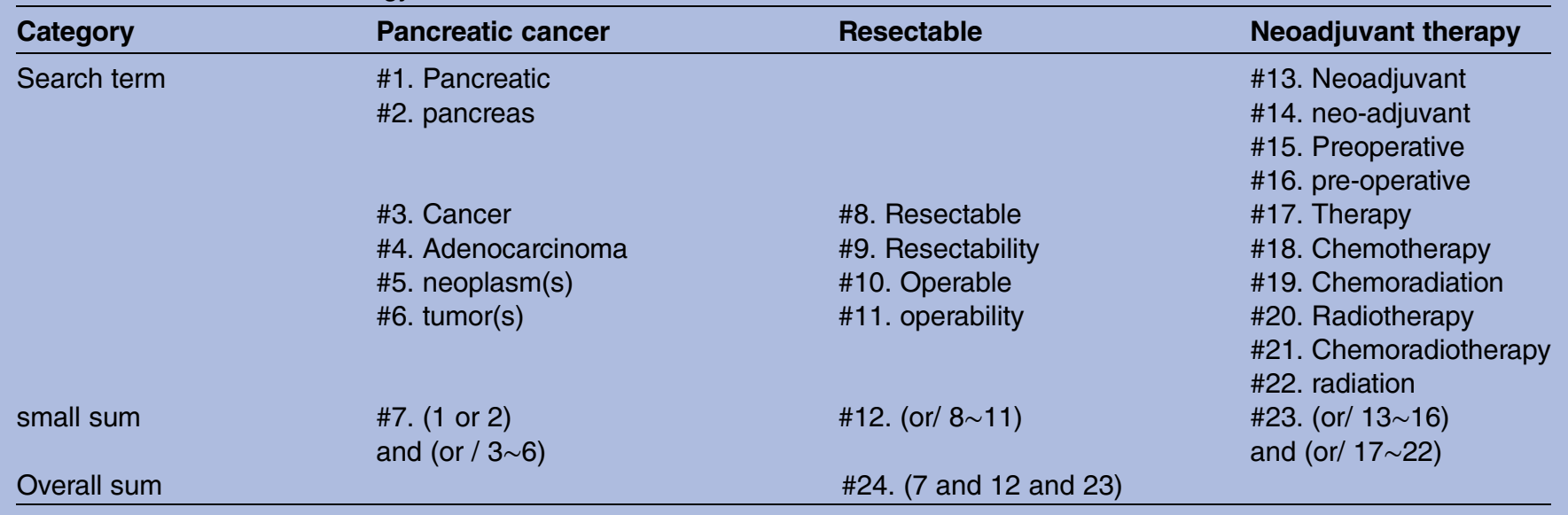


Table 3 Inclusion and exclusion criteria

\begin{tabular}{|c|c|c|c|}
\hline Exclusion step & The first exclusion (with title and abstract) & $\begin{array}{l}\text { The second exclusion } \\
\text { (with full text review) }\end{array}$ & Final inclusion \\
\hline Reasons & $\begin{array}{l}\text { 1. not in English } \\
\text { 2. not human study (preclinical, animal study) } \\
\text { 3. only for pediatric study } \\
\text { 4. not original article } \\
\text { 5. duplication check, 2nd }\end{array}$ & $\begin{array}{l}\text { 1. not adenoCa } \\
\text { 2. no resectable PC } \\
\text { 3. no neoadjuvant } \\
\text { 4. other neoadjuvant } \\
\text { 5. single-arm observation } \\
\text { 6. 5. duplication check, 3rd }\end{array}$ & $\begin{array}{l}\text { 1. English } \\
\text { 2. Human } \\
\text { 3. Adult } \\
\text { 4. original article } \\
\text { group I. RCTs } \\
\text { group II. Q-RCTs } \\
\text { NRCTs, PCSs }\end{array}$ \\
\hline $\begin{array}{l}\text { Corresponding } \\
\text { PRISMIA step }\end{array}$ & Screening $\rightarrow$ & Eligibility $\rightarrow$ & Included \\
\hline
\end{tabular}

\section{Assessment of risk of bias in included studies}

The risk of bias will be independently evaluated by two authors (JL and HK). The result of the assessment will also be verified by the methodologist (SA). Since this systematic review will include two groups (RCTs and NRS's), different assessment tools will be applied. First, the 'Risk of bias' tool of the Cochrane Handbook (V.5.1.0) will be used for group I studies. ${ }^{38}$ The studies will be categorised as 'high-risk', 'low-risk' or 'unclear risk' of bias. Similarly, 'NRS checklists' and NRSMG recommendation will be used in assessing group II studies. ${ }^{34} 35$ Disagreement between two authors will be resolved by a consensus meeting with $\mathrm{KP}$ and SA.

\section{Special consideration for NRS's in group II}

Since NRS's are more likely to be biased than RCTs, the authors will make special considerations for inclusion, assessment, synthesis and analysis of NRS's. One independent methodologist will play a leading role in every process of NRS review, and authors will establish a consensus about NRS issues. The basic framework of this chapter consists of study design, confounding, selective reporting and directness, using the recommendations of 'NRS checklists' from the Ottawa Non-Randomized Studies Workshop. ${ }^{34}$

\section{Study design}

- In screening NRS's, the authors will include Q-RCTs, NRCTs and PRS's in group II. However, this classification will not be dependent on the 'label' of a study but on the actual features of the full texts. The common data extraction form (DEF) will be used for RCTs and NRS's, and will contain the study type and reason for inclusion or exclusion (see online supplementary appendix 2).

- Citations will be triaged for eligibility according to the full text review. If there is any indication of a relevant comparison of neoadjuvant treatment and upfront surgery in the first step of screening, two authors (J-cL and HWK) will review the full text of the paper and decide on eligibility. The final decision on inclusion will be discussed in a consensus meeting of four authors (Jingu Kang, HWK, $\mathrm{K}-\mathrm{hP}$ and SA).

\section{Confounding}

- The likely domains of confounding and matched variables are as follows:

1. Confounding by indication: cancer staging, other malignancy, previous CTx or CRT.

2. Operational confounding: CTx regimen, CRT protocol, other major operation history.

3. Procedural confounding: not applicable, because this is not used for experimental studies.

4. Person confounding: age, sex, other comorbidity, performance status.

- Some probable association between the confounder and the outcome is expected. For example, patient age, performance status and cancer staging will be associated with OS and DFS in a linear-shaped curve. The likely direction of these associations is expected to be positive.

- With a full-text review, unmeasured and residual confounding will be verified by forms of the NRS checklist and NRSMG recommendations. ${ }^{34}{ }^{35}$ Since the DEF is a brief form for the first step of screening, a more detailed data selection sheet (DSS) will be used on the basis of Cochrane recommendations. ${ }^{39}$

\section{Selective reporting}

- Since many NRS's do not have detailed protocols, the risk of reporting biases such as selective outcome reporting and selective analysis reporting can be higher than for RCTs. A methodologist (SA) will identify these biases by modifying the framework of RCTs. ${ }^{40}$

- In the consensus meeting, the authors will discuss whether included studies have been designed on the basis of well-defined hypotheses relevant to our review questions, or on the basis of 'incidentally' arising questions. The results of the discussion will be applied to the assessment of each study. 


\section{Directness}

- As mentioned above, the purpose of the NRS's in group II in this systematic review is independent or parallel analysis, not complementary, sequential or replacement analysis, as in previous recommendations. ${ }^{41}$ The reasons for including NRS's in this systematic review are described in the Introduction section.

- All the PICO elements (population, interventions and comparators) of the primary studies in group II are matched to those in group I studies.

\section{Data extraction}

Two authors (JL and HK) will individually extract the data. The extraction process will be based on the DEF and DSS, which will be established by consensus meeting (JL, HK, KP and SA). We will extract the data from each included study as follows.

- Study characteristics: (1) study ID, (2) name of reviewer, (3) title, (4) author, (5) published year, (6) nation, (7) level of hospital, (8) sample size and (9) study design.

- Demographic characteristics: (1) age, with median and range, (2) sex as percentage of females, (3) cancer stage, (4) type of neoadjuvant therapy, (5) neoadjuvant CTx regimen or CRT protocol and (6) outcomes in each study.

- Check for exclusion: (1) other than English, (2) other than clinical trial, (3) only for paediatric population, (4) other than original article, (5) duplication, (6) pathology other than adenocarcinoma, (7) not including patients with RPC, (8) not including neoadjuvant CTx or CRT, (9) single-armed observational study and (10) vague category.

If the required data are ambiguous or not reported in the clinical articles, the authors will contact the first or corresponding author of the study by telephone or email and then collect the missing data using the DEF and DSS.

\section{Synthesis and analysis}

All statistical syntheses and analyses will be performed using Review Manager Software (V.5.3, Cochrane Collaboration, http://tech.cochrane.org/RevMan) and STATA for Windows (V.14.0, STATA Corp., Texas, USA). If more than two studies are eligible for analysis in each study type, we will combine the studies for meta-analysis by study types. Different study designs (group I, II) will be analysed separately.

\section{Effect size and pooled estimate (model)}

We will synthesise the HRs of OS and DFS. If the information from an individual study is insufficient, we will estimate overall HR from the studies using Tierney's method. ${ }^{42}$ This method will be applied only in group I studies.

Unlike previous meta-analyses, we will use the DerSimonian-Laird random-effects model to obtain the pooled estimate for groups I and II because the included studies will have an expectedly high heterogeneity due to different CTx regimens and CRT protocols. $^{4344}$

\section{Heterogeneity analysis}

Statistical heterogeneity will be visualised with forest plots in each group. The analysis of statistical heterogeneity among studies will be evaluated using $\mathrm{I}^{2}$-statistics, where values of $30 \%, 50 \%$ and $75 \%$ represent cut-off points for low, moderate and high degrees of heterogeneity, respectively. ${ }^{45}$ If there are any kinds of heterogeneity, subgroup analysis will be performed to determine the reason for heterogeneity. If some linear correlation between survival outcomes and a given covariate is suspected, we will consider a meta-regression in group I. ${ }^{46}$ If a sufficient number of RCTs are identified, subgroup analyses will be performed according to: (1) different types of neoadjuvant regimen, (2) dose and protocol of radiation, and (3) patient gender and age group.

\section{Publication bias}

Publication bias will be visualised with contour-enhanced funnel plots in each group. ${ }^{47}$ Egger's test will be performed in each study group to evaluate asymmetry in funnel plots. ${ }^{48}$ Cumulative meta-analysis will also be performed for group I studies because CTx regimens and CRT protocols have changed. ${ }^{49}$

\section{Sensitivity analysis}

Sensitivity analysis will be performed if significant heterogeneity still exists after robust subgroup analysis. The meta-analysis will be repeated after excluding lower quality studies according to suitable assessment tools in each group. ${ }^{34} 3538$ Then the results of the two meta-analyses will be compared. The authors will discuss and decide whether or not the lower quality studies should be excluded, depending on their strength of evidence, sample size and influence on the pooled estimate.

\section{Evaluation of the level of evidence}

The level of evidence will be evaluated with the GRADE (Grading of Recommendations Assessment, Development and Evaluation) system, using the GRADEpro program (V.3.6, GRADE working group, http://tech.cochrane.org/revman/gradepro). ${ }^{50}$ These tables will include a summary of the intervention effect and quality of individual outcomes using the GRADE approach. The quality of the body of evidence for each outcome will be assessed on the basis of five factors: study limitations, consistency of effect, imprecision, indirectness and publication bias.

\section{ETHICS AND DISSEMINATION}

This systematic review does not require formal ethical approval because the data of this analysis do not involve 
personal information and privacy. The findings of this systematic review and meta-analysis will provide a general overview and evidence of the effectiveness and safety of neoadjuvant therapy for OS and DFS. The findings will be disseminated through peer-reviewed publications or conference presentations.

\section{Acknowledgements The authors thank Cactus Communications for English proofreading}

Contributors $\mathrm{J}-\mathrm{CL}$ and $\mathrm{J}-\mathrm{HH}$ planned the protocol, JKa assisted in protocol design, J-HH and JKi provided clinical advice for the study protocol, K-hP revised the search strategy, J-CL, SA, and J-HH drafted the protocol, J-CL and HWK will search for studies and extract and analyse data, and SA has a critical role in reviewing NRS's.

\section{Competing interests None declared.}

Provenance and peer review Not commissioned; externally peer-reviewed.

Open Access This is an Open Access article distributed in accordance with the Creative Commons Attribution Non Commercial (CC BY-NC 4.0) license, which permits others to distribute, remix, adapt, build upon this work noncommercially, and license their derivative works on different terms, provided the original work is properly cited and the use is non-commercial. See: http:// creativecommons.org/licenses/by-nc/4.0/

\section{REFERENCES}

1. World Cancer Research Fund International. Pancreatic Cancer Statistics. 2012. http://www.wcrf.org/int/cancer-facts-figures/ data-specific-cancers/pancreatic-cancer-statistics

2. Siegel RL, Miller KD, Jemal A. Cancer statistics, 2015. CA Cancer J Clin 2015;65:5-29.

3. Ferlay J, Steliarova-Foucher E, Lortet-Tieulent J, et al. Cancer incidence and mortality patterns in Europe: estimates for 40 countries in 2012. Eur J Cancer 2013;49:1374-403.

4. De Angelis R, Sant M, Coleman MP, et al. Cancer survival in Europe 1999-2007 by country and age: results of EUROCARE-5-a population-based study. Lancet Oncol 2014;15:23-34.

5. Rahib L, Smith BD, Aizenberg R, et al. Projecting cancer incidence and deaths to 2030: the unexpected burden of thyroid, liver, and pancreas cancers in the United States. Cancer Res 2014;74:2913-21.

6. Oettle $\mathrm{H}$, Neuhaus $\mathrm{P}$, Hochhaus A, et al. Adjuvant chemotherapy with gemcitabine and long-term outcomes among patients with resected pancreatic cancer: the CONKO-001 randomized trial. JAMA 2013;310:1473-81.

7. Schnelldorfer T, Ware AL, Sarr MG, et al. Long-term survival after pancreatoduodenectomy for pancreatic adenocarcinoma: is cure possible? Ann Surg 2008;247:456-62.

8. Neoptolemos JP, Stocken DD, Friess $\mathrm{H}$, et al. A randomized trial of chemoradiotherapy and chemotherapy after resection of pancreatic cancer. N Engl J Med 2004;350:1200-10.

9. Neoptolemos JP, Stocken DD, Bassi C, et al. Adjuvant chemotherapy with fluorouracil plus folinic acid vs gemcitabine following pancreatic cancer resection: a randomized controlled trial. JAMA 2010;304:1073-81.

10. Maeda A, Boku N, Fukutomi A, et al. Randomized phase III trial of adjuvant chemotherapy with gemcitabine versus S-1 in patients with resected pancreatic cancer: Japan Adjuvant Study Group of Pancreatic Cancer (JASPAC-01). Jpn J Clin Oncol 2008;38:227-9.

11. Regine WF, Winter KA, Abrams RA, et al. Fluorouracil vs gemcitabine chemotherapy before and after fluorouracil-based chemoradiation following resection of pancreatic adenocarcinoma: a randomized controlled trial. JAMA 2008;299:1019-26.

12. Klinkenbijl JH, Jeekel J, Sahmoud T, et al. Adjuvant radiotherapy and 5-fluorouracil after curative resection of cancer of the pancreas and periampullary region: phase III trial of the EORTC gastrointestinal tract cancer cooperative group. Ann Surg 1999;230:776-82.

13. National Comprehensive Cancer Network (NCCN). Pancreatic Adenocarcinoma; Version 2.2015. http://www.nccn.org/professionals/ physician_gls/pdf/pancreatic.pdf

14. Seufferlein T, Bachet JB, Van Cutsem E, et al. Pancreatic adenocarcinoma: ESMO-ESDO Clinical Practice Guidelines for diagnosis, treatment and follow-up. Ann Oncol 2012;23(Suppl 7): vii33-40.

15. Heestand GM, Murphy JD, Lowy AM. Approach to patients with pancreatic cancer without detectable metastases. J Clin Oncol 2015;33:1770-8.

16. Sutton JM, Abbott DE. Neoadjuvant therapy for pancreas cancer: past lessons and future therapies. World J Gastroenterol 2014;20:15564-79.

17. Yeung RS, Weese JL, Hoffman JP, et al. Neoadjuvant chemoradiation in pancreatic and duodenal carcinoma. A Phase II Study. Cancer 1993;72:2124-33. http://dx.doi.org/10.1002/10970142(19931001)72:7<2124::AID-CNCR2820720711>3.0.CO;2-C

18. Heinrich S, Schäfer M, Weber A, et al. Neoadjuvant chemotherapy generates a significant tumor response in resectable pancreatic cancer without increasing morbidity: results of a prospective phase II trial. Ann Surg 2008;248:1014-22.

19. Tzeng CW, Tran Cao HS, Lee JE, et al. Treatment sequencing for resectable pancreatic cancer: influence of early metastases and surgical complications on multimodality therapy completion and survival. J Gastrointest Surg 2014;18:16-24; discussion 24-5.

20. Brunner TB, Scott-Brown M. The role of radiotherapy in multimodal treatment of pancreatic carcinoma. Radiat Oncol 2010;5:64.

21. Heinrich S, Pestalozzi BC, Schäfer M, et al. Prospective phase II trial of neoadjuvant chemotherapy with gemcitabine and cisplatin for resectable adenocarcinoma of the pancreatic head. $J$ Clin Oncol 2008;26:2526-31.

22. Lowy AM. Neoadjuvant therapy for pancreatic cancer. J Gastrointest Surg 2008;12:1600-8.

23. Ryan DP. Phase II Study of Preoperative FOLFIRINOX Versus Gemcitabine/Nab-Paclitaxel in Patients With Resectable Pancreatic Cancer. Clinicaltrial.gov 2014; NCT02243007.

24. Krause K, Ettrich T. Neoadjuvant Plus Adjuvant or Only Adjuvant Nab- Paclitaxel Plus Gemcitabine for Resectable Pancreatic Cancer (NEONAX). Clinicaltrial.gov 2014; NCT02047513.

25. Al-Batran SE, Pauligk C. Randomized Multicenter Phase II/III Study With Adjuvant Gemcitabine Versus Neoadjuvant / Adjuvant FOLFIRINOX for Resectable Pancreas Carcinoma. Clinicaltrial.gov 2014;NCT02172976.

26. Raoul JL. Neoadjuvant Gemcitabine/Oxaliplatin Plus Adjuvant Gemcitabine in Resectable PAncreatic Cancer (NEOPAC). Clinicaltrial.gov 2011;NCT01521702.

27. Golcher $\mathrm{H}$, Brunner TB, Witzigmann $\mathrm{H}$, et al. Neoadjuvant chemoradiation therapy with gemcitabine/cisplatin and surgery versus immediate surgery in resectable pancreatic cancer: results of the first prospective randomized phase II trial. Strahlenther Onkol 2015;191:7-16.

28. Casadei R, Di Marco M, Ricci C, et al. Neoadjuvant chemoradiotherapy and surgery versus surgery alone in resectable pancreatic cancer: a single-center prospective, randomized, controlled trial which failed to achieve accrual targets. J Gastrointest Surg 2015;19:1802-12.

29. Xu CP, Xue XJ, Liang N, et al. Effect of chemoradiotherapy and neoadjuvant chemoradiotherapy in resectable pancreatic cancer: a systematic review and meta-analysis. J Cancer Res Clin Oncol 2014;140:549-59.

30. Andriulli A, Festa V, Botteri E, et al. Neoadjuvant/preoperative gemcitabine for patients with localized pancreatic cancer: a meta-analysis of prospective studies. Ann Surg Oncol 2012;19: 1644-62.

31. Sharma G, Whang EE, Ruan DT, et al. Efficacy of neoadjuvant versus adjuvant therapy for resectable pancreatic adenocarcinoma: a decision analysis. Ann Surg Oncol 2015;22(Suppl 3):1229-37.

32. DE Felice F, Musio D, Raffetto N, et al. Neoadjuvant strategy as initial treatment in resectable pancreatic cancer: concrete evidence of benefit. Anticancer Res 2014;34:4673-6.

33. Wetterslev J, Thorlund K, Brok J, et al. Trial sequential analysis May establish when firm evidence is reached in cumulative meta-analysis. J Clin Epidemiol 2008;61:64-75.

34. Wells GA, Shea B, Higgins JP, et al. Checklists of methodological issues for review authors to consider when including non-randomized studies in systematic reviews. Res Synth Methods 2013;4:63-77.

35. Barnaby C, Reeves JJD, Higgins JP. Chapter 13: Including non-randomized studies. Cochrane Handbook. 2008. http://hiv. cochrane.org/sites/hiv.cochrane.org/files/uploads/Ch13_NRS.pdf

36. Moher D, Liberati A, Tetzlaff J, et al. Preferred reporting items for systematic reviews and meta-analyses: the PRISMA statement. Ann Intern Med 2009;151:264-9, w264.

37. U.S. department of health and human services. Common Terminology Criteria for Adverse Events (CTCAE) Version 4.03. National Institutes of Health, National Cancer Institute, 2010. 
http://evs.nci.nih.gov/ftp1/CTCAE/CTCAE_4.03_2010-06-14_Quick Reference_5x7.pdf

38. Higgins JP, Altman DG, Gøtzsche PC, et al. The Cochrane Collaboration's tool for assessing risk of bias in randomised trials. BMJ 2011;343:d5928.

39. Cochrane review group on HIV, AIDS. Data collection form: Intervention review-RCTs and non-RCTs. The Cochrane Collaboration, 2014. http://hiv.cochrane.org/sites/hiv.cochrane.org/ files/uploads/Data\%20extraction\%20form_all\%20studies.docx

40. Kirkham JJ, Dwan KM, Altman DG, et al. The impact of outcome reporting bias in randomised controlled trials on a cohort of systematic reviews. BMJ 2010;340:C365.

41. Schünemann HJ, Tugwell $P$, Reeves BC, et al. Non-randomized studies as a source of complementary, sequential or replacement evidence for randomized controlled trials in systematic reviews on the effects of interventions. Res Synth Methods 2013;4:49-62.

42. Tierney JF, Stewart LA, Ghersi D, et al. Practical methods for incorporating summary time-to-event data into meta-analysis. Trials 2007;8:16

43. DerSimonian R, Laird N. Meta-analysis in clinical trials. Control Clin Trials 1986;7:177-88.
44. Kontopantelis E, Reeves D. Performance of statistical methods for meta-analysis when true study effects are non-normally distributed: a comparison between DerSimonian-Laird and restricted maximum likelihood. Stat Methods Med Res 2012;21: 657-9.

45. Higgins JP, Thompson SG. Quantifying heterogeneity in a meta-analysis. Stat Med 2002;21:1539-58.

46. Simmonds MC, Higgins JP. A general framework for the use of logistic regression models in meta-analysis. Stat Methods Med Res 2014. Published Online First 12 May 2014.

47. Peters JL, Sutton AJ, Jones DR, et al. Contour-enhanced meta-analysis funnel plots help distinguish publication bias from other causes of asymmetry. J Clin Epidemiol 2008;61:991-6.

48. Egger M, Davey Smith G, Schneider M, et al. Bias in meta-analysis detected by a simple, graphical test. BMJ 1997;315:629-34.

49. Lau J, Schmid CH, Chalmers TC. Cumulative meta-analysis of clinical trials builds evidence for exemplary medical care. J Clin Epidemiol 1995;48:45-57; discussion 59-60.

50. Cochrane Informatics \& Knowledge Management Department. GRADEpro 3.6 program. Cochrane collaboration, 2013. http://tech. cochrane.org/revman/gradepro 